\title{
Expression Levels of miR-30c and miR-186 in Adult Patients with Membranous Glomerulonephritis and Focal Segmental Glomerulosclerosis
}

This article was published in the following Dove Press journal: International Journal of Nephrology and Renovascular Disease

\author{
Seyyedeh Mina Hejazian ${ }^{1-3}$ \\ Mohammadreza Ardalan ${ }^{2}$ \\ Mohammadali M Shoja ${ }^{4}$ \\ Nasser Samadi' \\ Sepideh Zununi Vahed (1D) ${ }^{2}$ \\ 'Department of Medical Biotechnology, \\ Faculty of Advanced Medical Sciences, \\ Tabriz University of Medical Sciences, \\ Tabriz, Iran; ${ }^{2}$ Kidney Research Center, \\ Tabriz University of Medical Sciences, \\ Tabriz, Iran; ${ }^{3}$ Student Research \\ Committee, Tabriz University of Medical \\ Sciences, Tabriz, Iran; ${ }^{4}$ Department of \\ Surgery, University of Illinois at Chicago- \\ Metropolitan Group Hospitals (UIC- \\ MGH), Chicago, IL, USA
}

Correspondence: Sepideh Zununi Vahed Kidney Research Center, Tabriz University of Medical Sciences, Tabriz, Iran

Email sepide.zununi@gmail.com
Background: Nephrotic syndrome is a common renal problem with different histopathogenesis. MicroRNAs are reported to be involved in the pathophysiology of the syndrome. The aim of this study was to study the levels of miR-30c and miR-186 in NS patients.

Methods: Sixty patients with primary NS (membranous glomerulonephritis (MGN, N=30) and focal segmental glomerulosclerosis (FSGS, N=30)) and 24 healthy volunteers were included. Expression levels of the miR-30c and miR-186 were evaluated in plasma and peripheral blood mononuclear cell (PBMC) samples of adult patients with NS using real-time PCR. Moreover, an in-silico analysis was performed to understand the signaling pathways and biological procedures that may be regulated by these miRNAs.

Results: In the MGN group, significantly elevated levels of miR-30c and miR-186 were observed in PBMC $(\mathrm{P}=0.037)$ and plasma $(\mathrm{P}=0.035)$ samples, respectively. Moreover, there was a significant increase in miR-30c levels in PBMC samples of the FSGS group when compared to healthy controls $(\mathrm{P}=0.004)$. In ROC curve analysis, combined levels of the studied miRNAs could discriminate cases from controls in plasma and blood cells (AUC $\geq 0.72, \mathrm{P}<0.05$ ).

Conclusion: A panel of miRNAs may be potential biomarkers in plasma and PBMCs samples of NS patients with different subclasses. More investigations are needed with a large sample size to validate the diagnostic values of the reported miRNAs.

Keywords: nephrotic syndrome, proteinuria, membranous glomerulonephritis, focal segmental glomerulosclerosis, microRNAs, biomarker

\section{Introduction}

Nephrotic syndrome (NS) is a common renal problem defined by proteinuria, edema, and hypoalbuminemia. ${ }^{1,2}$ In adults, NS can be caused by different glomerular diseases, including Minimal Change Nephropathy (MCN), Focal Segmental Glomerulosclerosis (FSGS), and Membranous Glomerulonephritis (MGN). In this respect, each type of NS has its specific pathogenesis, for instance, podocytes, specialized kidney glomerular cells, injury is a major cause of FSGS and MCN, while deposited antibodies against phospholipase A2 receptor (PLA2R) on podocytes are the main cause of adult MGN. ${ }^{3-5}$ The common clinical method for NS diagnosis is renal biopsy that is an invasive procedure. $^{6}$ Therefore, it is essential to define the underlying histopathological diagnosis with a non-invasive and more reliable method to employ a specific therapy and monitor response to therapy. 
MicroRNAs are small non-coding ribonucleotides that are involved in different cell procedures by binding to $3^{\prime}$ UTR (untranslated-region) region of mRNAs and silencing their expression at both transcriptional and translational levels. $^{7,8}$ About $30 \%$ of mRNAs are regulated by all 3000 identified human microRNAs. ${ }^{9}$ Based on the stability and function of miRNAs in cells and body fluids and their expression changes during various diseases, they can be considered as possible diagnostic and prognostic biomarkers. ${ }^{10-13}$ In recent studies, altered expression of multiple miRNAs and their target genes are reported in different kidney diseases. ${ }^{14-16}$ Likewise, a specific set of miRNAs is described to be deregulated in different biological sources of children and adults with podocyte injury. Although these previous reports offer some insights into the impact of miRNAs in podocytopathy, more efforts need to be done to understand the clinical significance values of the miRNAs involved in the pathophysiology of NS.

Based on the controversial results obtained from different clinical sources of NS patients, ${ }^{17-21}$ there is a lack of definite early non-invasive biomarkers for NS diagnosis. It was the aim of the present study to assess the levels of circulating and cellular levels of miR-30c-5p and miR186 involved in podocyte homeostasis and podocytopathies in blood samples of adults with NS and to determine their potential diagnostic or prognostic values.

\section{Materials and Methods}

\section{Study Subjects}

The present cross-sectional study recruited 60 primary NS patients from the Nephrology Ward of Imam Reza Hospital (Tabriz, Iran). Blood samples were collected from November 2017 to March 2019. Nephrotic proteinuria was defined by excretion of protein $\geq 3 \mathrm{~g} / 24 \mathrm{~h}$ in urine, as heavy proteinuria. Inclusion criteria for cases were the age range between 20 and 60, primary NS, and normal urinary system without any obstruction. Patients with secondary NS causes such as lupus, diabetes, amyloidosis, viral infection, and drug-related NS were not included. The exclusion criteria for cases were inflammatory diseases, diabetes, a history of cancer, severe bacterial infection, serologic positivity for CMV, HBV, HIV, HCV and BK viruses, obstruction in the renal system, overlap syndrome, any other glomerulopathy, severe organ failure, autoimmune disorder and those with ESRD under dialysis. Histologic detail of patients was collected from the renal biopsy findings. Further, a group of healthy volunteers $(n=24)$ with no clinical history of kidney disease was also allocated as controls. Inclusion criteria for normal controls were matched-age and -gender with the cases and having normal kidney function (normal serum creatinine and urine analysis).

\section{RNA Extraction from Plasma and PBMC}

Fresh blood samples $(6-8 \mathrm{~mL})$ were collected in EDTAcoated Vacutainer tubes and all processes were performed within 2 hours in the laboratory. For preparing plasma samples, $2 \mathrm{~mL}$ of blood sample centrifuged at $1000 \times \mathrm{g}$ for 10 minutes two times at $4^{\circ} \mathrm{C}$. Then, supernatant (plasma) was aliquot in microtubes and stored at $-70^{\circ} \mathrm{C}$ for future uses. For peripheral blood mononuclear cells (PBMC) isolation, $4 \mathrm{~mL}$ of peripheral blood was diluted with an equal volume of phosphate-buffered saline (PBS) solution and then added into $4 \mathrm{~mL}$ Ficoll-Paque. The solution centrifuged at $800 \times \mathrm{g}$ for 20 minutes. PBMCs layer between plasma and Ficoll was taken and transferred into a new RNase-free $15 \mathrm{~mL}$ falcon. PBMCs washed with $10 \mathrm{~mL}$ PBS and centrifuged at $500 \times \mathrm{g}$ for 10 minutes. The supernatant removed and the pellet was washed with $1 \mathrm{~mL}$ PBS again and centrifuged at $500 \times \mathrm{g}$ for 10 minutes. Finally, pellet solved in $1 \mathrm{~mL}$ Trizol and stored at $-70^{\circ} \mathrm{C}$ for later use. Before RNA isolation from plasma samples $(250 \mu \mathrm{L}), 3.5 \mu \mathrm{L}$ cel-miR-39 miRNeasy Serum/Plasma Spike-In $\left(1.6 \times 10^{8}\right.$ copies $\left./ \mu \mathrm{L}\right)$ (Qiagen, Germany) was added to all samples as exogenous control. The total RNA extraction was performed based on the Trizol protocol (RiboEx $^{\mathrm{TM}}$, South Korea). Optical Density (OD) was calculated for the detection of RNA integrity, quality, and quantity via A260/A280 and A230/A280 nm by NanoDrop (NanoDrop $^{\text {TM }}$ One $^{\mathrm{C}}$ Thermo Scientific, USA).

\section{cDNA Synthesis and Quantitative Real-Time PCR (Q-PCR)}

Synthesis of the first strand of cDNA in both plasma and PBMCs samples was performed separately in $15 \mu \mathrm{L}$ reaction volume; RNA ( $5 \mu \mathrm{L}$ RNA extracted from plasma and $1 \mu \mathrm{g}$ RNA extracted from PBMCs), $0.8 \mu \mathrm{L}$ RT enzyme, $3 \mu \mathrm{L}$ RT buffer, $0.375 \mu \mathrm{L}$ Ribolock, $1.5 \mu \mathrm{L}$ dNTP, $1.325 \mu \mathrm{L}$ DEPC, and $3 \mu \mathrm{L}$ primer mix (including equal volumes of customized stemloop primers of miR-30c, miR-186, U6, spiked in cel-miR-39, Snord-47 and UR (universal reverse)) Supplementary Table S1. Then, cDNA $(0.5 \mu \mathrm{L})$ amplified in a reaction of $10 \mu \mathrm{L}$ volume using $2 \mu \mathrm{L}$ master mix $\left(5 \times\right.$ HOT FIREPol ${ }^{\circledR}$ 
EvaGreen ${ }^{\circledR}$ qPCR Mix Plus, Biodyne, Austria), $0.5 \mu \mathrm{L}$ forward primer, $0.5 \mu \mathrm{L}$ reverse primer, and $6.5 \mu \mathrm{L}$ DEPC. For each sample, a duplicate run was done. The qPCR was performed at $95^{\circ} \mathrm{C}$ for $15 \mathrm{~min}$, followed by 40 cycles each at $95^{\circ} \mathrm{C}$ for $10 \mathrm{~s}$, and annealing at $60^{\circ} \mathrm{C}$ for $30 \mathrm{~s}$. Melting curves were made at $72-95^{\circ} \mathrm{C}$ to verify the specificity of reaction after the completion of PCR cycling. Cel-miR-39, SNORD-47, and U6 were used as internal controls for plasma and PBMCs, respectively. The gene expression was calculated with the $2^{-\Delta \Delta \mathrm{Ct}}$ method.

\section{Statistical Analysis}

To test the normal distribution of variables, the ShapiroWilk test was used. Categorical variables presented by numbers and percentages. Mean \pm standard deviation (SD) and median (Max-Min) were used for normally and nonnormally distributed variables, respectively. For comparisons of quantitative variables between two groups, Student's $t$-test or Mann-Whitney $U$-tests were used while the chi-squared test was used for categorical variables. One-way ANOVA test with Tukey's HSD post hoc test was used for normally and Kruskal-Wallis was used for non-normally distributed variables between more than two groups. Spearman correlation was used for the evaluation of correlations between variables. The receiver operating characteristic (ROC) curve analysis was used for evaluating the potential of each miRNA in discriminating cases from controls or for discriminating cases with FSGS from MGN. The optimal threshold was obtained by Youden's method. Statistical analyses were performed by the IBM SPSS 17.0 Software (SPSS, Inc.). $\mathrm{P}<0.05$ was considered statistically significant.

\section{In-Silico Analysis}

An in-silico analysis was performed to understand the underlying signaling pathways and biological procedures regulated by the studied miRNAs. Using miRTarBase 6.0 database (Available at http://mirtarbase.mbc.nctu.edu.tw/php/index. php), a list of the confirmed targets of hsa-miR-30c and hsamiR-186 were obtained and subjected to the ORA (overrepresentation enrichment analysis) based on Kyoto Encyclopedia of Genes and Genomes (KEGG) and gene ontology (GO) pathways using the WebGestalt web server (Available at http://www.webgestalt.org/option.php). In these analyses, the reference gene list was set to "genome_protein_coding" and the multiple test adjustment method set to Benjamini-Hochberg (BH). Other factors set as default. The enrichment ratio (R) was considered for each significant category that stands for the ratio of observed versus expected number of genes. Besides, a literature search was made to find the functions of target genes that may be relevant to NS.

\section{Results \\ Subjects}

Sixty patients with NS, 30 patients with biopsy-proven FSGS, and 30 patients with positive anti-PLA2 receptor antibody and biopsy-proven MGN were included in this study. Table 1 summarizes the clinical and demographic data of patients. Regardless of differences in their histopathology, all of the patients were associated with features of the primary NS and podocyte injury. Most of the patients were refractory to the treatment and they were failed to achieve remission of their proteinuria after the treatment period. There were no significant differences in age and sex between cases and controls $(\mathrm{P}=0.54)$. Moreover, no significant differences detected in age, gender, GFR or serum levels of creatinine, uric acid, urea and $24 \mathrm{~h}$ urine creatinine between the different subtypes of cases ( $\mathrm{P}>0.05)$ (Table 1).

Among the dysregulated miRNAs in NS, the expression levels of miR-30c and miR-186 were evaluated in both plasma and PBMCs specimens of the cases by q-PCR. Plasma samples were used for the analysis of miRNAs since the true profiling of circulating miRNAs may be altered during the coagulation procedure in serum preparation. Despite significant reported values of exogenous control, neither spiked-in cel-miR-39-5p nor $-3 p$ was appropriate for analysis of data; therefore, U6 used as an internal control for normalization of circulating miRNAs in plasma samples. Moreover, PBMCs were used since the evaluation of miRNAs in these cells had not been reported in patients with NS.

Statistically, there was an increase in miR-30c level in the peripheral cells of the NS $(\mathrm{P}=0.005), \mathrm{MGN}(\mathrm{P}=0.037)$, and FSGS ( $\mathrm{P}=0.004)$ patients when compared to controls. Plasma levels of miR-30c were not significant between the studied groups, Figure $1 \mathrm{~A}$ and $\mathrm{B}$. Circulating $(\mathrm{P}=0.045)$ but not cellular $(\mathrm{P}=0.70)$ levels of miR-186 increased in NS groups significantly when compared to controls (Figure 1C and D). Additionally, the increased level of circulating miR-186 was significantly obvious in the MGN group $(\mathrm{P}=0.035)$ but not in the FSGS group $(\mathrm{P}=0.183)$ as compared to controls. However, increased levels of PBMCs' miR-186 were not statistically significant in the MGN group in comparison to controls $(\mathrm{P}=0.91)$ and FSGS cases $(\mathrm{P}=0.28)$, Figure $1 \mathrm{C}$ and $\mathrm{D}$.

There were correlations between the studied miRNAs and other clinical variables in NS patients; however, the differences were not statistically significant (data not 
shown). There were significant correlations between the studied miRNAs levels in plasma and PBMC (Table 2).

\section{ROC Analysis}

To further examine the values of the studied miRNAs to discriminate subtypes of NS, the ROC analysis was done. Fold change values were used to compute the ROC curve. ROC results are presented in Table 3 with more details. PBMC levels of miR-30c showed a sensitivity of $46 \%$ and specificity of $96 \%$ with $\mathrm{AUC}=0.65,95 \% \mathrm{CI} ; 0.565$ to 0.728 to discriminate NS from controls. Circulating miR186 with $\mathrm{AUC}=0.61,82 \%$ sensitivity, and $43 \%$ specificity could discriminate FSGS from MGN cases.

The combined effect of two circulating miRNAs with $\mathrm{AUC}=0.72 \quad[0.64-0.82] \quad(\mathrm{p}<0.001), 86 \%$ sensitivity, and $51 \%$ specificity could distinguish NS patients from healthy controls $(\mathrm{PPV}=0.806[0.715$ to 0.874$], \mathrm{NPV}=0.613[0.438$ to 0.763 ], $\mathrm{LR}^{+}=1.77$ [1.259 to 2.493], and $\mathrm{LR}^{-}=0.268$ [0.146 to 0.495]). Cellular levels of these miRNAs with $\mathrm{AUC}=0.72$ [0.62-0.81] $(\mathrm{p}<0.001), 53 \%$ sensitivity and $89 \%$ specificity could distinguish NS patients from healthy controls with $\mathrm{PPV}=0.915[0.801$ to 0.966$], \mathrm{NPV}=0.471[0.359$ to 0.587$]$, $\mathrm{LR}^{+}=4.98[1.928$ to 12.824$], \mathrm{LR}^{-}=0.518[0.399$ to 0.674$]$. Moreover, with $\mathrm{AUC}=0.69$ and $\mathrm{AUC}=0.71$, PBMCs levels of the studied miRNAs could discriminate FSGS from MGN, respectively. Although each of the studied miRNAs alone could not discriminate the individuals in the studied groups, their combined levels showed a reliable moderate accuracy. However, both high sensitivity and specificity were not observed (Figure 2A-D).

\section{In-Silico Analysis}

The ORA identified biological processes that are overrepresented among target genes of miR-30c and miR-186 (Table 4).
GO and KEGG enrichment analysis proposed that the predicted targets may participate in a variety of physiological regulatory functions in NS. This result shows that miR-30c by targeting genes involved in the chromatin modification, gene expression, double-strand RNA (dsRNA) and mRNA metabolic processes, cell cycle and posttranscriptional regulation of gene expression may be involved in NS. Additionally, miR-186 predicted to target chromosome organization, RNA splicing process, gene transcription and translation, peptide biosynthetic, and cellular macromolecule catabolic processes. Moreover, 10 common pathways in the KEGG pathway were found for the studied miRNAs (Supplementary Tables S2 and $\underline{\mathrm{S} 3}$ ). A literature search indicated that some target genes of miR-30c and miR-186 may be functionally related to NS.

\section{Discussion}

To validate the potential value of miRNAs involved in the pathogenesis of NS, the expression levels of miR-30c and miR-186 in plasma and PBMCs samples were compared between NS cases and healthy individuals. The result indicated that PBMCs level of miR-30c was upregulated significantly in NS group compared to healthy groups. Moreover, there was a significant difference in miR-30c levels between different pathology subtypes of NS in comparison to control group. Furthermore, this study demonstrated a significant increase in plasma (not PBMCs) level of miR-186 levels in the MGN group when compared to controls. The combined levels of the studied miRNAs in plasma and PBMCs could discriminate NS patients from controls.

MiR-30 family is a significant epigenetic regulator in podocyte homeostasis and their production can be changed in the course of NS. Evidence shows that the level of miR30 family is altered with NS progression and therapy. ${ }^{22,23}$ In

Table I Demographic and Baseline Clinical Data

\begin{tabular}{|c|c|c|c|c|}
\hline Characteristics & Total NS Patients & MGN & FSGS Group & P-value ${ }^{a}$ \\
\hline No. of cases & 60 & 30 & 30 & \\
\hline Age, mean $\pm S D$ (years) & $45.76 \pm 13$ & $46.6 \pm 13$ & $44.5 \pm 12.8$ & 0.404 \\
\hline BMI $\left(\mathrm{kg} / \mathrm{m}^{2}\right)$ & $27.3 \pm 3.4$ & $27.4 \pm 3.6$ & $27.3 \pm 3.4$ & 0.49 \\
\hline $\operatorname{GFR}(\mathrm{mL} / \mathrm{min} / 1.73 \mathrm{~m})$ & $80(10-137)$ & $80(18-137)$ & $79(10-130)$ & $0.324^{\mathrm{b}}$ \\
\hline Proteinuria (mg/24 h) & $2497(85-15,900)$ & $3013(85-15,000)$ & $1360(97-\mid 5,900)$ & $0.26^{\mathrm{b}}$ \\
\hline Urea (mg/dL) & $40.7 \pm 19.4$ & $38.2 \pm 16.5$ & $44.2 \pm 20$ & 0.18 \\
\hline Uric acid (mg/dL) & $6.5 \pm 1.1$ & $6.61 \pm 1.2$ & $6.3 \pm 1.1$ & 0.94 \\
\hline Serum creatinine $(\mathrm{mg} / \mathrm{dL})$ & $1.2 \pm 0.5$ & $2.21 \pm 0.55$ & $1.2 \pm 0.5$ & 0.17 \\
\hline Urine creatinine $(\mathrm{mg} / 24 \mathrm{~h})$ & I | $24.38 \pm 407$ & || $59.2 \mid \pm 328$ & $1074.63 \pm 500$ & 0.742 \\
\hline
\end{tabular}

Notes: GFR: estimated glomerular filtration rate as measured by GFR $\left[186 \times(\text { Creatinine/88.4 })^{-1.154} \times(\text { Age })^{-0.203} \times(0.742\right.$ if female $\left.)\right]$ formula. The quantity data are expressed as mean \pm SD. ${ }^{a} M G N$ versus FSGS. ${ }^{b}$ Median (Min-Max) is presented. P-value is based on Mann-Whitney U-test. 

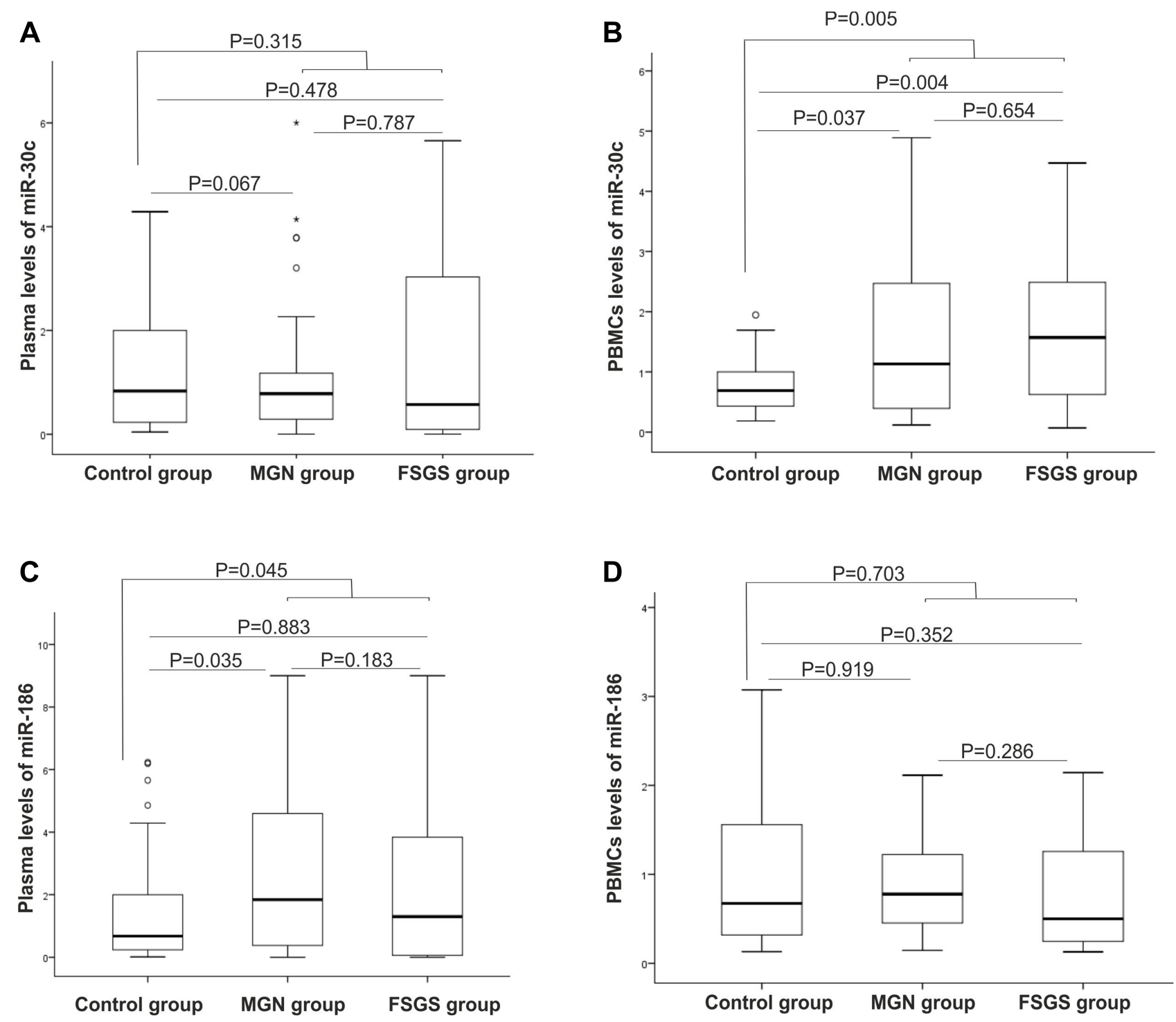

Figure I The expression of miRNA-30c-5p and miRNA-186-5p in plasma and PBMCs. The expression of miRNA-30c-5p in (A) Plasma and (B) PBMCs between the studied groups. The expression of miRNA-186-5p in (C) Plasma and (D) PBMCs between the studied groups. The relative expression (fold change) was calculated by the $2^{-\Delta \Delta C t}$ method. $*$ and $\circ$ are outliers.

Abbreviations: NS, nephrotic syndrome; FSGS, focal segmental glomerulosclerosis; MGN, membranous glomerulonephritis.

serum and urine samples, ${ }^{23}$ and urinary exosomes ${ }^{24}$ of children with active NS, increased concentration of miR-30a-5p was observed, while its level tended to decrease with the remission of patients. In contrast to these results, downregulated levels of miR-30 family were reported in podocytes of FSGS cases $^{17}$ and kidney tissue of children with FSGS but not $\mathrm{MGN}^{21}$ in comparison to normal controls. Furthermore, the high throughput sequencing of microRNAs showed that miRNA-30 family was downregulated in peripheral blood lymphocyte cells (PBLCs) of MGN patients in comparison to healthy individuals. ${ }^{22}$ Higher plasma levels of miR-30c were also found in MCN cases compared to FSGS patients and controls; however, there were no significant differences in miR-30c level between FSGS and healthy controls. ${ }^{18}$ In the present study, the PBMCs level of miR-30c significantly elevated in the NS group when compared to healthy controls. Moreover, there was a statistically significant change in the FSGS and MGN groups when compared to controls.

Elevated and diminished levels of miR-186 have been described in previous studies in NS patients. ${ }^{20,21}$ In this research, an overexpression was observed in the circulating miR-186 level in the NS group and MGN. Additionally, no statistically significant changes were observed between the NS group and controls or among 
Table 2 Internal Correlations Between miRNA Levels in Plasma and Peripheral Blood Cells

\begin{tabular}{|c|c|c|c|c|}
\hline & $\begin{array}{l}\text { Plasma } \\
\text { miR-30c }\end{array}$ & $\begin{array}{l}\text { Plasma } \\
\text { miR-I } 86\end{array}$ & $\begin{array}{l}\text { PBMCs } \\
\text { miR-30c }\end{array}$ & $\begin{array}{l}\text { PBMCs } \\
\text { miR- I } 86\end{array}$ \\
\hline Plasma & I & $r=0.779 *$ & $r=-0.191$ & $r=-0.099$ \\
\hline $\mathrm{miR}-30 \mathrm{c}$ & & $p<0.001$ & $p=0.137$ & $p=0.449$ \\
\hline Plasma & & 1 & $r=-0.176$ & $r=-0.141$ \\
\hline miR-I 86 & & & $\mathrm{p}=0.167$ & $\mathrm{P}=0.273$ \\
\hline PBMCs & & & I & $r=0.60^{*}$ \\
\hline miR-30c & & & & $P<0.001$ \\
\hline
\end{tabular}

Note: *Correlation is significant.

Abbreviation: $r$, correlation coefficient.

FGSG and MGN groups in PBMC samples. In a literature review, elevated levels of miR-186 are reported in plasma samples of patients with active FSGS relative to healthy controls; however, miR-186 level remained unchanged in steroid-resistant patients before and after steroid treatment. ${ }^{20}$ It should be also noted that the plasma level of miR-186 was not increased in groups of MGN and diabetic nephropathy patients with nephrotic proteinuria. ${ }^{20}$ However, downregulated levels of miR-186 were observed both in human podocytes cell line and renal biopsy of MGN patients. $^{21}$ These results showed that miR-186 might not be involved in the pathogenesis of FSGS.

ROC analysis indicated that each of the studied miRNAs did not have strong power to discriminate NS patients from controls and differentiate histological subtypes of NS in our study. However, their combined levels could distinguish NS patients from healthy control and also discriminate subtypes of NS with either reliable sensitivity or specificity. In our study, there was a positive strong association between miR-30c and miR-186 in both PBMCs and plasma samples of NS patients, but not between plasma and PBMCs samples.

All these studies along with the present work indicated that levels of microRNA are dysregulated in NS patients with different histopathology; however, the data are not consistent. Several reasons may be accounted for the differences between miRNA levels in different reports, including patients' related factors (age, stage of the disease, genetic background), sample size, different ethnic population, different sample sources (serum, plasma, PBMCs, PBLCs, podocyte, and kidney biopsy), and the method of miRNAs evaluation (use of different internal control, control group). Moreover, treatment strategy (the type of drugs and course of treatment at the time of sampling) would be another possible explanation for divergent miRNA expression.

Besides, we performed a bioinformatics analysis to identify the possible targets of the studied genes in the pathogenesis of NS. MiR-30s can guard podocytes against apoptosis by targeting p53 and Notch1 and the loss of miR-30s eases podocyte damage. Moreover, miR-30s stabilize the podocyte cytoskeletons by targeting some other genes. ${ }^{17}$ Additionally, miR-30s can regulate calcineurin/calcium signaling in podocytes. $^{25}$ Overexpression of miR-30c could reduce Notch1 protein levels in the podocytes. ${ }^{17}$ Our bioinformatics analysis along with the literature review predicted that miR-30 family may participate in the pathogenesis of NS by targeting different genes involved in TGF- $\beta$, Notch1, and p53 signaling pathways. It is also reported that the TGF- $\beta 1$ pathway has an important role in the pathogenesis of FSGS. $^{26-28}$ The reduced number of podocytes induced by

Table 3 Results of the ROC Curve Analysis for Discriminating Potential of PBMC and Plasma miR-30c and miR-I86 Levels Among Pairs of the Studied Groups

\begin{tabular}{|c|l|l|l|l|l|l|l|}
\hline & AUC & $\mathbf{9 5 \%}$ Cl & P-value & Sensitivity & Specificity & Youden Index J & Associated Criterion \\
\hline NS and controls & & & & & & & \\
Plasma FC30c & 0.50 & 0.418 to 0.585 & 0.97 & 87 & 30 & 0.13 & $>0.02$ \\
Plasma FCI86 & 0.53 & 0.419 to 0.591 & 0.92 & 54 & 29 & 0.16 & $\leq 2.58$ \\
PBMC FC30c & 0.65 & 0.565 to 0.728 & 0.001 & 46 & 96 & 0.4158 & $>2$ \\
PBMC FCI86 & 0.45 & 0.418 to 0.582 & 1 & 0 & 100 & 0 & $>0$ \\
\hline FSGS and MGN & & & & & & & \\
Plasma FC30c & 0.52 & 0.421 to 0.621 & 0.72 & 65 & 54 & 0.1849 & $>0.66$ \\
Plasma FCI86 & 0.61 & 0.499 to 0.707 & 0.07 & 82 & 43 & 0.2472 & $>0.19$ \\
PBMC FC30c & 0.54 & 0.437 to 0.647 & 0.48 & 89 & 30 & 0.1902 & $\leq 3.56$ \\
PBMC FCI86 & 0.5 & 0.400 to 0.600 & 0.0 & 0 & 100 & 0 & $>0$ \\
\hline
\end{tabular}

Abbreviations: AUC, area under the curve; $\mathrm{Cl}$, confidence interval; ROC, receiver operating characteristic; NS, nephrotic syndrome; MGN, membranous glomerulonephritis; FSGS, focal segmental glomerulosclerosis; FC, fold change; PFC, plasma FC. 
A

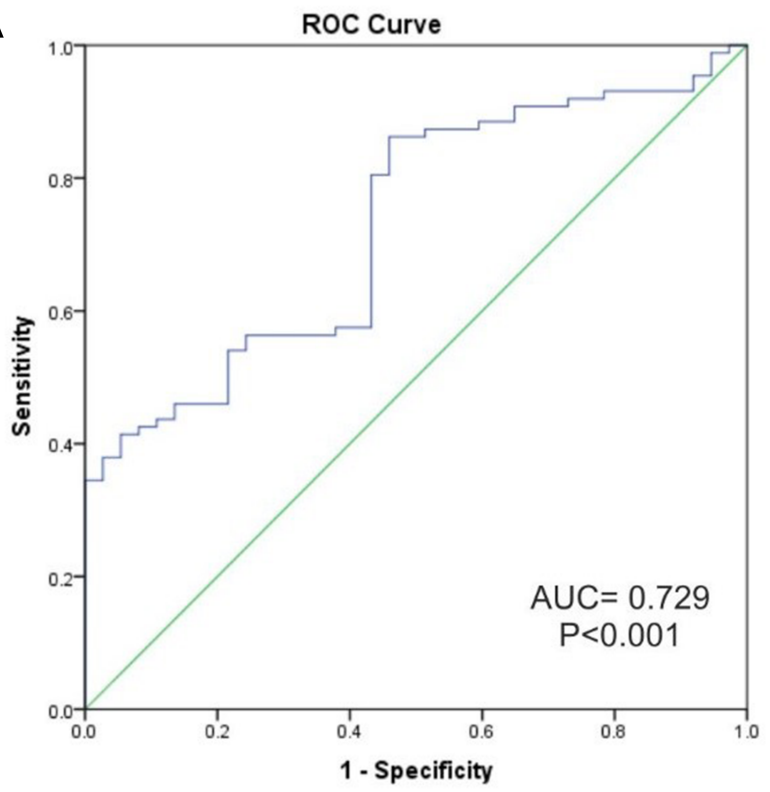

C

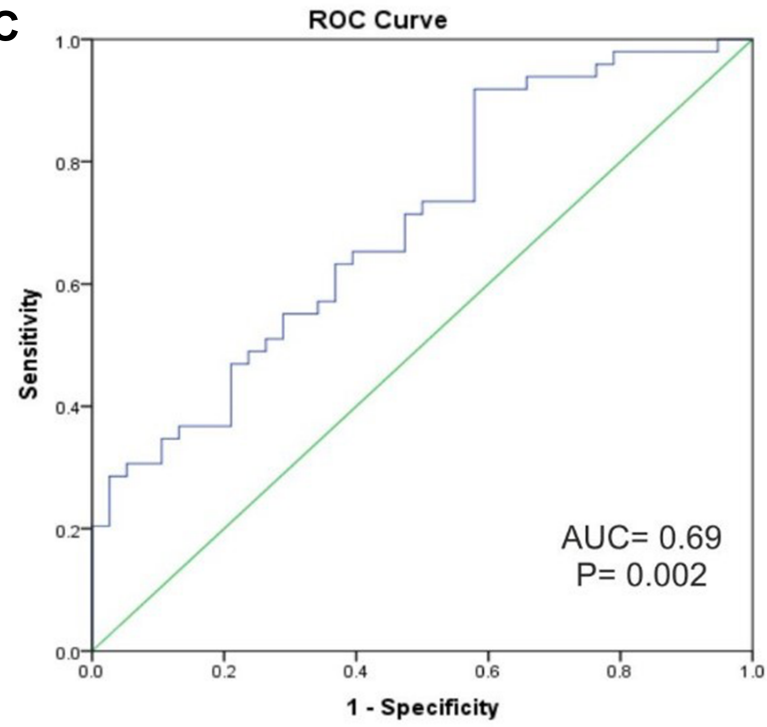

B

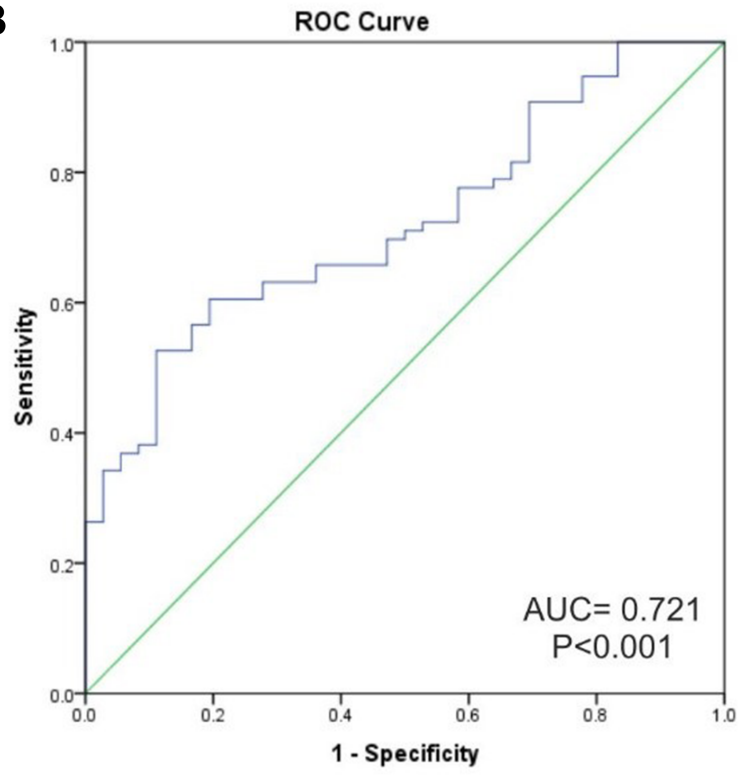

D

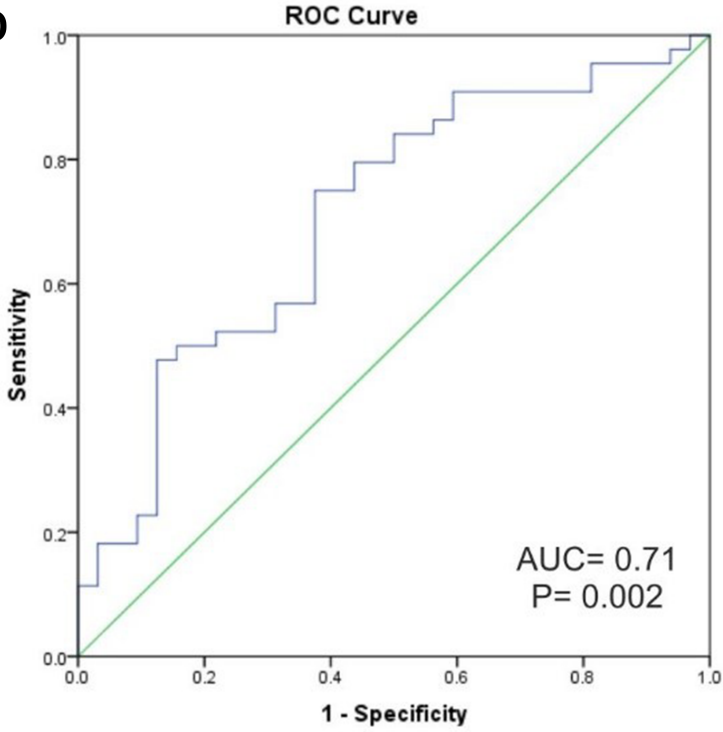

Figure 2 The combined ROC curves analysis of miR-30c and miR-186 in plasma and PBMCs samples. (A) Plasma and (B) PBMCs levels of the studied miRNAs could discriminate NS patients from controls. (C, D) ROC curve for the potential of the studied miRNAs in discriminating FSGS from MGN patients in (C) Plasma and (D) PBMCs. AUCs and the corresponding statistics of each miRNA between the groups are shown in Table 3.

Abbreviations: ROC, receiver operating characteristic; AUC, area under the curve.

apoptosis has a serious role in the development of MGN and decreased levels of miR-186 leads to an increase in its target genes Toll-like receptor 4 (TLR4), caspase-3, and P2X7 that are involved in apoptosis ${ }^{21}$ and may contribute to the development of FSGS.

A small sample size was the possible limitation of the present study. Different results from biological resources were considered as another limitation. We used strict criteria for the collection of the patients to validate the reported candidate miRNAs and to lessen potential unclear factors on the miRNAs expression. Since NS (especially FSGS subtype) has heterogeneous pathogenesis, ${ }^{28}$ it is difficult to identify an ideal biomarker for specific pathology of NS in all cases. This heterogeneity needs to study a large cohort to validate the reported microRNAs as diagnostic and prognostic biomarkers in patients with different subclasses of NS. Additionally, a potential diagnostic/prognostic value of miRNAs in MGN and FSGS requires further studies. 
Table 4 The Ten Top Enriched Biological Processes Among Target Genes of miR-30c and miR-186

\begin{tabular}{|c|c|c|c|c|c|c|}
\hline GO ID & Biological Process & C & 0 & $\mathbf{E}$ & $\mathbf{R}$ & p-value \\
\hline \multicolumn{7}{|l|}{ miR-30c } \\
\hline GO:007067I & Response to interleukin- 12 & 49 & 9 & 1.518943743 & 5.925170068 & $1.67 \mathrm{E}^{-05}$ \\
\hline GO:0040029 & Regulation of gene expression, epigenetic & 258 & 22 & 7.997703789 & 2.750789549 & $1.82 \mathrm{E}^{-05}$ \\
\hline GO:000I763 & Morphogenesis of a branching structure & 196 & 18 & $6.07577497 \mid$ & 2.962585034 & $3.96 \mathrm{E}^{-05}$ \\
\hline GO:0002009 & Morphogenesis of an epithelium & 480 & 32 & $|4.8794489|$ & 2.150617284 & $4.08 \mathrm{E}^{-05}$ \\
\hline GO:0045023 & G0 to $\mathrm{GI}$ transition & 45 & 8 & 1.394948335 & 5.734979424 & $6.30 \mathrm{E}^{-05}$ \\
\hline GO:0031050 & dsRNA processing & 48 & 8 & $|.487,944,89|$ & 5.37654321 & $\mathrm{I} .02 \mathrm{E}^{-04}$ \\
\hline GO:0016569 & Covalent chromatin modification & 468 & 30 & 14.50746269 & 2.067901235 & $1.43 \mathrm{E}^{-04}$ \\
\hline GO:0104004 & Cellular response to environmental stimulus & 320 & 23 & 9.919632606 & 2.318634259 & $1.67 \mathrm{E}^{-04}$ \\
\hline GO:I,990,823 & Response to leukemia inhibitory factor & 95 & 11 & 2.94489093 & 3.735282651 & $1.69 \mathrm{E}^{-04}$ \\
\hline GO:0010608 & Posttranscriptional regulation of gene expression & 486 & 30 & 15.06544202 & 1.9913123 & $2.74 \mathrm{E}^{-04}$ \\
\hline \multicolumn{7}{|l|}{ miR-186 } \\
\hline GO:001607I & mRNA metabolic process & 765 & 79 & 30.80383 & 2.564616 & $8.88 \mathrm{E}^{-15}$ \\
\hline GO:0010629 & Negative regulation of gene expression & 1733 & 127 & 69.78175 & 1.81996 & $1.0 I \mathrm{E}^{-11}$ \\
\hline GO:00064I2 & Translation & 613 & 62 & 24.68333 & 2.511817 & $2.06 \mathrm{E}^{-11}$ \\
\hline GO:0006396 & RNA processing & 887 & 78 & 35.71633 & 2.183875 & $5.1 I E^{-11}$ \\
\hline GO:0043043 & Peptide biosynthetic process & 636 & 62 & 25.60946 & 2.420981 & $9.58 \mathrm{E}^{-11}$ \\
\hline GO:005I 276 & Chromosome organization & 1143 & 92 & 46.02454 & 1.998933 & $1.00 \mathrm{E}^{-10}$ \\
\hline GO:0044265 & Cellular macromolecule catabolic process & 1108 & 88 & 44.61522 & 1.972421 & $5.32 \mathrm{E}^{-10}$ \\
\hline GO:0008380 & RNA splicing & 417 & 46 & $|6.79| 1 \mid$ & 2.739545 & $5.62 \mathrm{E}^{-10}$ \\
\hline GO:0043604 & Amide biosynthetic process & 766 & 68 & $30.844 I$ & 2.204636 & $6.64 \mathrm{E}^{-10}$ \\
\hline GO:0006397 & mRNA processing & 487 & 50 & 19.60976 & $2.54975 ।$ & $1.20 \mathrm{E}^{-09}$ \\
\hline
\end{tabular}

Note: ${ }^{\text {a}}$ The Benjamini-Hochberg adjusted $p$-value.

Abbreviations: $\mathrm{C}$, total number of genes in the category; $\mathrm{O}$, number of observed target genes in the category; $\mathrm{E}$, number of expected genes in the category; $\mathrm{R}$, fold enrichment.

\section{Conclusion}

We investigated the expression levels of miR-30c and miR-186 in PBMC and plasma samples of patients with NS. There were significant differences between the NS group and its subtypes when compared to controls. The combined levels of the studied miRNAs could distinguish NS patients from healthy control with a moderate accuracy. Further investigations are essential to validate a panel of miRNAs with diagnostic and prognostic values in NS patients with different histopathology.

\section{Ethical Statement}

This study was conducted in accordance with the Declaration of Helsinki. This study was certified by the Ethics Committee of Tabriz University of Medical Sciences, Tabriz, Iran (IR.TBZMED.REC.1396.912). By providing written informed consent, subjects agreed to participate in this study.

\section{Acknowledgment}

The authors gratefully thank to Faculty of Advanced Medical Sciences and Department of Biochemistry at
Tabriz University of Medical Sciences for their technical support. The authors wish to express their gratitude toward the Nephrology Ward of Imam-Reza Hospital for their honest assistance in sampling. We also want to express special gratitude to Dr. Milad Bastami and Dr. Ziba Nariman-Saleh-Fam for designing the primers and performing bioinformatics analysis.

\section{Author Contributions}

All authors made a significant contribution to the work reported, whether that is in the conception, study design, execution, acquisition of data, analysis and interpretation, or in all these areas; took part in drafting, revising or critically reviewing the article; gave final approval of the version to be published; have agreed on the journal to which the article has been submitted; and agree to be accountable for all aspects of the work.

\section{Funding}

This work was financially supported by the Kidney Research Center at Tabriz University of Medical Sciences, Tabriz, Iran (MSc. Thesis No: 96/2-3/13). 


\section{Disclosure}

The authors declared no conflicts of interest.

\section{References}

1. Macé C, Chugh SS. Nephrotic syndrome: components, connections, and angiopoietin-like 4-related therapeutics. J Am Soc Nephrol. 2014;25(11):2393-2398. doi:10.1681/ASN.2014030267

2. Lee SJ, Borsting E, Declèves A-E, Singh P, Cunard R. Podocytes express IL-6 and lipocalin 2/neutrophil gelatinase-associated lipocalin in lipopolysaccharide-induced acute glomerular injury. Nephron Exp Nephrol. 2012;121:e86-e96. doi:10.1159/000345151

3. de Seigneux S, Martin PY. Management of patients with nephrotic syndrome. Swiss Med Wkly. 2009;139:416-422. doi:10.4414/ smw.2009.12477

4. Ronco P, Debiec H. Pathogenesis of membranous nephropathy: recent advances and future challenges. Nat Rev Nephrol. 2012;8 (4):203. doi:10.1038/nrneph.2012.35

5. Bierzynska A, Soderquest K, Koziell A. Genes and podocytes - new insights into mechanisms of podocytopathy. Front Endocrinol (Lausanne). 2015;5:226. doi:10.3389/fendo.2014.00226

6. Xiao B, Wang L-N, Li W, et al. Plasma microRNA panel is a novel biomarker for focal segmental glomerulosclerosis and associated with podocyte apoptosis. Cell Death Dis. 2018;9(5):533. doi:10.1038/ s41419-018-0569-y

7. Gebeshuber CA, Kornauth C, Dong L, et al. Focal segmental glomerulosclerosis is induced by microRNA-193a and its downregulation of WT1. Nat Med. 2013;19(4):481-487. doi:10.1038/nm.3142

8. Inui M, Martello G, Piccolo S. MicroRNA control of signal transduction. Nat Rev Mol Cell Biol. 2010;11(4):252-263. doi: $10.1038 / \mathrm{nrm} 2868$

9. Huttenhofer A, Mayer G. Circulating miRNAs as biomarkers of kidney disease. Clin Kidney J. 2017;10(1):27-29. doi:10.1093/ckj/ sfw075

10. Jones MR, Quinton LJ, Blahna MT, et al. Zcchc11-dependent uridylation of microRNA directs cytokine expression. Nat Cell Biol. 2009;11(9):1157. doi:10.1038/ncb1931

11. Katoh T, Sakaguchi Y, Miyauchi K, et al. Selective stabilization of mammalian microRNAs by $3^{\text {' }}$ adenylation mediated by the cytoplasmic poly(A) polymerase GLD-2. Genes Dev. 2009;23(4):433-438. doi:10.1101/gad.1761509

12. Mitchell PS, Parkin RK, Kroh EM, et al. Circulating microRNAs as stable blood-based markers for cancer detection. Proc Natl Acad Sci. 2008;105(30):10513-10518. doi:10.1073/pnas.0804549105

13. Chen Y, Gelfond JA, McManus LM, Shireman PK. Reproducibility of quantitative RT-PCR array in miRNA expression profiling and comparison with microarray analysis. BMC Genomics. 2009;10 (1):407. doi:10.1186/1471-2164-10-407

14. Ho J, Kreidberg JA. The long and short of MicroRNAs in the kidney: figure 1. J Am Soc Nephrol. 2012;23(3):400-404. doi:10.1681/ ASN.2011080797
15. Nariman-Saleh-Fam Z, Bastami M, Ardalan M, Sharifi S, Hosseinian Khatib SM, Zununi Vahed S. Cell-free microRNA-148a is associated with renal allograft dysfunction: implication for biomarker discovery. $J$ Cell Biochem. 2019;120:5737-5746. doi:10.1002/jcb.27860

16. Vahed SZ, Zonouzi AP, Mahmoodpoor F, et al. Circulating miR-150, miR-192, miR-200b, and miR-423-3p as non-invasive biomarkers of chronic allograft dysfunction. Arch Med Res. 2017; 48(1): 96-104. doi: 10.1016/j.arcmed.2017.03.004

17. Wu J, Zheng C, Fan Y, et al. Downregulation of MicroRNA-30 facilitates podocyte injury and is prevented by glucocorticoids. J Am Soc Nephrol. 2014;25(1):92-104. doi:10.1681/ASN.2012111101

18. Ramezani A, Devaney JM, Cohen S, et al. Circulating and urinary microRNA profile in focal segmental glomerulosclerosis: a pilot study. Eur J Clin Invest. 2015;45(4):394-404. doi:10.1111/eci.12420

19. Shi S, Yu L, Chiu C, et al. Podocyte-selective deletion of dicer induces proteinuria and glomerulosclerosis. J Am Soc Nephrol. 2008;19(11):2159. doi:10.1681/ASN.2008030312

20. Zhang C, Zhang W, Chen HM, et al. Plasma MicroRNA-186 and in focal segmental proteinuria glomerulosclerosis. Am J Kidney Dis. 2014;65:223-232. doi:10.1053/j.ajkd.2014.07.013

21. Sha W-G, Shen L, Zhou L, Xu D-Y, Lu G-Y. Down-regulation of miR-186 contributes to podocytes apoptosis in membranous nephropathy. Biomed Pharmacother. 2015;75:179-184. doi:10.1016/ j.biopha.2015.07.021

22. Zhang W, Zhang C, Chen H, et al. Evaluation of MicroRNAs miR-196a, miR-30a-5P, and miR-490 as biomarkers of disease activity among patients with FSGS. Clin J Am Soc Nephrol. 2014;9 (9):1545-1552. doi:10.2215/CJN.11561113

23. Yang Luo CW, Guan X, Chen X, et al. Increased serum and urinary MicroRNAs in children with idiopathic nephrotic syndrome. Clin Chem. 2013. doi:10.1373/clinchem.2012.195297

24. Chen $\mathrm{T}$, Wang $\mathrm{C}$, $\mathrm{Yu} \mathrm{H}$, et al. Increased urinary exosomal microRNAs in children with idiopathic nephrotic syndrome. EBioMedicine. 2019;39:552-561. doi:10.1016/j.ebiom.2018.11.018

25. Wu J, Zheng C, Wang X, et al. MicroRNA-30 family members regulate calcium/calcineurin signaling in podocytes. $J$ Clin Invest. 2015;125(11):4091-4106. doi:10.1172/jci81061

26. Bennett MR, Czech KA, Arend LJ, Witte DP, Devarajan P, Potter SS. Laser capture microdissection-microarray analysis of focal segmental glomerulosclerosis glomeruli. Nephron Exp Nephrol. 2007;107(1): e30-e40. doi:10.1159/000106775

27. Tong J, Xie J, Ren H, et al. Comparison of glomerular transcriptome profiles of adult-onset steroid sensitive focal segmental glomerulosclerosis and minimal change disease. PLoS One. 2015;10(11): e0140453. doi:10.1371/journal.pone.0140453

28. Schwab K, Witte DP, Aronow BJ, Devarajan P, Potter SS, Patterson LT. Microarray analysis of focal segmental glomerulosclerosis. Am J Nephrol. 2004;24(4):438-447. doi:10.1159/000080188
The International Journal of Nephrology and Renovascular Disease is an international, peer-reviewed open-access journal focusing on the pathophysiology of the kidney and vascular supply. Epidemiology, screening, diagnosis, and treatment interventions are covered as well as basic science, biochemical and immunological studies. The manuscript management system is completely online and includes a very quick and fair peer-review system, which is all easy to use. Visit http://www.dovepress.com/testimonials.php to read real quotes from published authors. 